\title{
Contingency is crucial for creating imitative responses
}

\section{Catmur*}

Economic and Social Research Council Centre for Economic Learning and Social Evolution, Department of Psychology, University College London, London, UK

${ }^{*}$ Correspondence: c.catmur@ucl.ac.uk

\section{A commentary on}

Acquisition of automatic imitation is sensitive to sensorimotor contingency

by Cook, R., Press, C., Dickinson, A., and Heyes, C. (2010). J. Exp. Psychol. Hum. Percept. Perform. 36, 840-852.

In order to imitate, we must translate the visual representation of an action into the motor commands which will produce the same action. A growing body of evidence suggests that the mirror system - comprising brain areas active during both the observation and the performance of actions - is involved in this process (Heiser et al., 2003; Catmur et al., 2009). It has also been suggested that, by representing another person's action in one's own motor system, the mirror system underlies not only imitation but also action understanding (Rizzolatti and Craighero, 2004). A similar self-other matching system may produce empathic responses to others' emotions. Now, a new study (Cook et al., 2010) has provided the first evidence to distinguish between the predictions of two competing theories of how mirror responses are acquired, and has demonstrated the key role of sensorimotor contingency.

How do mirror responses arise? Over the last 5 years it has become clear that mirror system responses to others' actions depend on experience, and in particular on sensorimotor experience, where the same action is observed and performed at the same time (Calvo-Merino et al., 2006; Catmur et al., 2007). Two contrasting theories have been proposed to explain how such experience can produce mirror responses. Heyes' Associative Sequence Learning (ASL) model (Heyes and Ray, 2000; Heyes, 2001) suggests that general processes of associative learning, such as those which support instrumental and Pavlovian conditioning, are sufficient to produce mirror responses. Alternatively, the Hebbian perspective (Keysers and Perrett, 2004) considers mirror responses to result from Hebbian learning.
These two accounts are similar in many ways, but differ in particular on one important point. Hebbian learning, as illustrated in the axiom "cells that fire together, wire together," relies on the temporal contiguity between stimuli and responses (observed and performed actions): any two representations which are active at the same time can become associated. Thus, under the Hebbian account, as long as they occur together, any observed action could become associated with any performed action. This has led to suggestions that the Hebbian account cannot fully explain why most mirror neurons respond to the observation and performance of the same action. Keysers and colleagues have therefore suggested that sensorimotor experience during development must be "canalized" to provide the right kind of matching input to the mirror system (del Giudice et al., 2009). In contrast, associative learning relies not only on contiguity but on contingency: the extent to which a stimulus is a reliable predictor of a response. The ASL theory therefore predicts that observed and performed actions will become associated only when a contingent, predictive relationship exists between the sight and the performance of an action.

A new study is the first to distinguish between the predictions of these two theories, by measuring the learning of counter-imitative responses. Cook et al. (2010) utilized the well-established finding that counter-mirror sensorimotor training (where participants see one action but perform another) leads to a reduction in subsequent imitation of the trained actions (Heyes et al., 2005). Cook et al. compared two groups, both of which received counter-mirror sensorimotor training: when they observed an opening hand, they closed their hand, and vice versa. The contiguity between observed and performed actions was the same in both groups: both received an equal number of sensorimotor pairings (see open, do close; see close, do open). However, in one group the contingency between observed and performed actions was perfect: the performance of a hand closing movement was always predicted by the observation of a hand opening movement. The second, non-contingent, group in addition performed an equal number of hand opening and closing movements which were not predicted by any observed movement. Thus, in this group, the contingency between observation of a movement and performance of a non-matching, counter-mirror, movement was zero.

Recall that learning in this experiment is indexed by a reduction in imitation of the trained actions, due to the counter-mirror sensorimotor training received. Cook et al. found that the group receiving contingent experience showed less imitation - i.e., more counter-mirror learning - than the non-contingent group. This was despite equal levels of contiguity in both groups. A follow-up experiment demonstrated that the effect of contingency could not be due to habituation resulting from the greater number of hand movements performed by the non-contingent group. This study therefore shows that the learning of imitative responses depends on both contiguity and contingency.

The study by Cook et al. used imitation as an index of mirror system activity. This is consistent with the widely held assumption that imitation is mediated by the mirror system (Heiser et al., 2003; Iacoboni, 2009), and with evidence that performance on an imitation task of the kind used by Cook et al. is disrupted by repetitive transcranial magnetic stimulation of the inferior frontal gyrus, a classical mirror area (Catmur et al., 2009). However, to confirm that it is not just the development of imitation, but also the development of mirror neurons, that depends on contingency as well as contiguity, it would be desirable to repeat the experiment using neurophysiological measures of mirror system activity.

Cook et al.'s paper answers the criticisms that an associative account of mirror system responses cannot explain their 
specificity: that an associative mechanism associates everything with everything else. Instead, the predictions resulting from this study are clear: mirror responses will develop for those actions for which the learner receives predictive, contingent sensorimotor experience, in which the observation of an action is reliably paired with its performance. These results also explain the existence of complementary mirror responses (Newman-Norlund et al., 2007), for example where the observation of someone releasing an object prompts the observer to grasp it: such responses will develop where the learner experiences a contingent relationship between seeing a release and performing a grasp.

Finally, by demonstrating that general processes of associative learning underlie the acquisition of mirror responses, this study implies that interventions based upon associative learning principles should be the most effective way of altering or improving mirror system responses.

\section{ACKNOWLEDGMENT}

The author is supported by the Economic and Social Research Council (grant no. RES-538-28-1001).

\section{REFERENCES}

Calvo-Merino, B., Grezes, J., Glaser, D. E., Passingham, R. E., and Haggard, P. (2006). Seeing or doing? Influence of visual and motor familiarity in action observation. Curr. Biol. 16, 1905-1910.

Catmur, C., Walsh, V., and Heyes, C. (2007). Sensorimotor learning configures the human mirror system. Curr. Biol. 17, 1527-1531.

Catmur, C., Walsh, V., and Heyes, C. (2009). Associative sequence learning: the role of experience in the development of imitation and the mirror system. Philos. Trans. R. Soc. Lond. B Biol. Sci. 364, 2369-2380.

Cook, R., Press, C., Dickinson, A., and Heyes, C. (2010). Acquisition of automatic imitation is sensitive to sensorimotor contingency. J. Exp. Psychol. Hum. Percept. Perform. 36, 840-852.

del Giudice, M., Manera, V., and Keysers, C. (2009). Programmed to learn? The ontogeny of mirror neurons. Dev. Sci. 12, 350-363.

Heiser, M., Iacoboni, M., Maeda, F., Marcus, J., and Mazziotta, J. C. (2003). The essential role of Broca's area in imitation. Eur. J. Neurosci. 17, 1123-1128.

Heyes, C. (2001). Causes and consequences of imitation. Trends Cogn. Sci. 5, 253-261.
Heyes, C., Bird, G., Johnson, H., and Haggard, P. (2005). Experience modulates automatic imitation. Brain Res Cogn. Brain Res. 22, 233-240.

Heyes, C. M., and Ray, E. D. (2000). What is the significance of imitation in animals? Adv. Stud. Behav. 29, 215-245.

Iacoboni, M. (2009). Imitation, empathy, and mirror neurons. Annu. Rev. Psychol. 60, 653-670.

Keysers, C., and Perrett, D. I. (2004). Demystifying social cognition: a Hebbian perspective. Trends Cogn. Sci. 8, 501-507.

Newman-Norlund, R. D., van Schie, H. T., van Zuijlen, A. M., and Bekkering, H. (2007). The mirror neuron system is more active during complementary compared with imitative action. Nat. Neurosci. 10, 817-818.

Rizzolatti, G., and Craighero, L. (2004). The mirrorneuron system. Annu. Rev. Neurosci. 27, 169-192.

Received: 20 September 2010; accepted: 24 January 2011; published online: 03 February 2011.

Citation: Catmur C (2011) Contingency is crucial for creating imitative responses. Front. Hum. Neurosci. 5:15. doi: 10.3389/fnhum.2011.00015

Copyright (c) 2011 Catmur. This is an open-access article subject to an exclusive license agreement between the authors and Frontiers Media SA, which permits unrestricted use, distribution, and reproduction in any medium, provided the original authors and source are credited. 